\title{
Determinación por Visión Artificial del Factor de Degradación en Aleaciones Biocompatibles
}

\author{
Willian Aperador-Chaparro ${ }^{(1)}$, Jorge H. Bautista-Ruiz ${ }^{(2)}$, Aura S. Mejía ${ }^{(3)}$ \\ (1) Departamento de Ingeniería, Universidad Militar Nueva Granada, Carrera 11 No. 101-80, \\ Bogotá-Colombia (e-mail:g.ing.materiales@gmail.com) \\ (2) Departamento de Física, Universidad Francisco de Paula Santander. Avenida Gran Colombia No 12E - \\ 96. B Colsag. Edificio de Laboratorios. San José de Cúcuta-Colombia (e-mail: jorgebautista@ufps.edu.co) \\ (3)Facultad de Ciencias, Escuela Colombiana de Ingeniería "Julio Garavito", AK.45 No.205-59 (Autopista \\ Norte), Bogotá-Colombia (e-mail: aura.mejia@escuelaing.edu.co)
}

Recibido Sep. 28, 2012; Aceptado Nov. 23, 2012; Versión final recibida Dic. 02, 2012

\section{Resumen}

Se determinó por visión artificial el factor de degradación de una aleación biocompatible, AISI 316LVM. Para ello, se utilizó una solución fisiológica simulada (solución de Hanks), electrolito que simula la composición presente en el organismo, es decir, el ambiente donde el implante se utilizará. El comportamiento electroquímico fue evaluado mediante curvas potencio-dinámicas. La caracterización superficial se desarrolló mediante un estereoscopio y los productos de corrosión se evaluaron mediante difracción de rayos $\mathrm{X}$. El sistema usó una imagen microscópica de la superficie del material en su estado natural (brillo espejo) como parámetro base para la comparación, para definir en qué estado se encuentran las muestras una vez han pasado por las pruebas realizadas. Se encontró, que es posible estimar el factor de degradación o de deterioro en un material mediante un análisis topográfico del mismo.

Palabras clave: biomateriales, degradación, visión artificial, 316LVM

\section{Computer Vision for Determination of Degradation Factor in Biocompatible Alloys}

\begin{abstract}
The degradation factor of a biocompatible alloy, AISI 316LVM, was determined by computer vision. For this, a simulated physiological solution (Hanks' solution) which simulates the electrolyte composition in the body, that is the environment in which the implant is used. The electrochemical behavior was evaluated by potentio-dynamic curves. The surface characterization was performed using a stereoscope and corrosion products were evaluated by X-ray diffraction. The system used a microscopic image of the surface of the material in its natural state (mirror finish) as a basis for comparison parameter to define what state are once samples have undergone testing. It was found that it is possible to estimate the factor of degradation or deterioration of a material through a topographic analysis.
\end{abstract}




\section{INTRODUCCIÓN}

La utilización de metales en implantes biomédicos ortopédicos y odontológicos se basa fundamentalmente en las solicitaciones mecánicas extremas a las que éstos están sometidos en servicio (Park, 1984; López, 2004). Idealmente, un implante metálico debería ser completamente inerte en el cuerpo humano; sin embargo, rara vez sucede así. Los fluidos orgánicos son extremadamente hostiles a los materiales metálicos y su efecto sobre los implantes y de éstos sobre los tejidos circundantes, es de gran interés científico. Los materiales metálicos empleados en implantes tienen tendencia termodinámica a corroerse; aunque, poseen en común la formación de una película protectora que es capaz de mantener los efectos de la corrosión en valores aceptables, y evitar que los productos de corrosión pueden resultar tóxicos para los tejidos. La eficacia de la película superficial depende de la resistencia de las capas de pasivación a la ruptura y de la capacidad de repasivación de los materiales en este medio de trabajo (López, 2004).

El uso de metales en cirugía ortopédica está a su vez condicionado por la agresividad del medio fisiológico y puede originar la liberación de productos de degradación y/o desgaste no deseados en el organismo (Jacobs, 1999; Venugopalan, 2001; Woodman, 1983). A pesar de los numerosos avances realizados en cirugía ortopédica, las soluciones aceptadas distan mucho de ser perfectas, en especial en cuanto al material se refiere. Los metales puros no tienen las propiedades que requieren los diferentes tipos de implantes utilizados actualmente en traumatología y ortopedia, como es el caso del titanio puro, dado que su uso está restringido debido a sus limitadas propiedades mecánicas. Por tal motivo, es necesario recurrir a la adición de uno o más metales al elemento base, para modificar su estructura cristalina y en consecuencia sus propiedades físicas y químicas. Un ejemplo de ello es la aleación Ti-6Al-4V, a pesar de que en los últimos años se ha desencadenado una cierta controversia sobre su biocompatibilidad (Bordji, 1996; Geetha, 2009; Tapash, 2011).

Los aceros inoxidables, son mayoritariamente utilizados a nivel mundial en implantes temporarios, mientras que las aleaciones de $\mathrm{Cr}$-Co y el $\mathrm{Ti}$ aleado se utilizan para implantes permanentes. La necesidad de reducción de costos en los servicios de salud pública ha redundado en el uso masivo del acero inoxidable como la opción más económica dentro de las aleaciones metálicas usadas en cirugía ortopédica (Samuel, 2008; De Mello, 2006).

El acero inoxidable AISI 316LVM es utilizado para aplicaciones médicas por estar fundido bajo vacío, lo cual genera un alto grado de pureza. Ofrece una excelente resistencia a tejidos y fluidos fisiológicos, a la corrosión intergranular y a la corrosión en general. Sin embargo, su utilización como biomaterial es cuestionada por su contenido de níquel, debido a la intolerancia a dicho metal que presentan algunas personas. Una desventaja potencial del acero inoxidable, en aplicaciones de prótesis, es su susceptibilidad a la corrosión por tensión. El agrietamiento por corrosión bajo tensión ocurre a bajas tensiones, situación frecuente en implantes con tensiones residuales: el inicio del agrietamiento es acelerado por el proceso de corrosión y las grietas siguen creciendo bajo las tensiones aplicadas. La preocupación sobre la corrosión y los subsecuentes efectos sobre la biocompatibilidad a largos plazos, ha motivado el empleo del acero inoxidable en sistemas de fijación de fracturas. Estas aplicaciones frecuentemente requieren la remoción del dispositivo al momento que ocurra la cura del hueso (Cook, 1986; Pourbaix, 1984).

La biocompatibilidad de un material comprende todas las reacciones y efectos que tienen lugar entre el implante y el cuerpo humano. La fase inicial del contacto está asociada con la interacción con un fluido, ya sea sangre, saliva o fluidos extracelulares, produciéndose una adsorción de macromoléculas desde el fluido hacia la superficie del implante. En este proceso, normalmente intervienen proteínas, la adsorción de las cuales juega un papel crucial en el mecanismo de biocompatibilidad, dado que está ligada con las interacciones directas que se producen en la interfase. Por una parte, el cuerpo humano ejerce una influencia sobre el material provocando un cambio en sus características, principalmente debido a procesos de corrosión y degradación, y por otra, la presencia del material provoca cambios en los tejidos circundantes a través de procesos de inflamación (Bonfiel, 1981; Samuel, 2008; Tapash, 2011).

En los implantes quirúrgicos, la corrosión puede ser un fenómeno crítico, que afecte tanto a la biocompatibilidad del implante como a la integridad estructural de la prótesis. La corrosión y la disolución de las capas superficiales del material son dos mecanismos que pueden derivar en la introducción de iones metálicos en el cuerpo humano, originando efectos adversos por reacción biológica de los mismos (BouSaleh, 2007; Brune, 1985).

En los últimos años, los sistemas de visión artificial han evolucionado tanto tecnológicamente como en la propia filosofía del sistema de visión. Esto ha implicado cambios sustanciales en la forma de interpretar la visión como una herramienta Standard para el análisis de procesos (Infaimon, 2010). La visión por computador, también denominada visión artificial, puede definirse como el proceso de extracción de 
información del mundo físico a partir de imágenes capturadas y transferidas a un computador. La información o tareas que un sistema de visión artificial puede realizar van desde la simple detección de objetos sencillos en la imagen, hasta la interpretación tridimensional de escenas complicadas (Rojas, 2008).

La visión artificial es una tecnología sofisticada de inspección de uso común en diversas industrias, por ejemplo en la acuacultura (Zion, 2012), la horticultura (Moreda, 2012) y es aplicable en la determinación de la cantidad de grasa en aves de corral (Chmiel, 2011). La visión artificial la componen un conjunto de procesos destinados a realizar el análisis de imágenes. Estos procesos son: captación de imágenes, memorización de la información, procesado e interpretación de los resultados. Es posible citar algunas aplicaciones de la visión artificial, como por ejemplo: automatizar tareas repetitivas de inspección realizadas por operadores, realizar controles de la calidad de productos que no era posible verificar por métodos tradicionales, realizar inspecciones de objetos sin contacto físico y reducir el tiempo de ciclo en procesos automatizados (Dutta, 2012; German, 2012)

El propósito del artículo será presentar la aplicación de la visión artificial para determinar el factor de degradación en la vida útil del acero 316LVM, sometido a condiciones de trabajo agresivos en una solución fisiológica simulada y aireada a pH 7,25 y $37^{\circ} \mathrm{C}$, contrastar los resultados con los obtenidos mediante la caracterización electroquímica y la identificación de los productos de corrosión (DRX).

\section{MATERIALES Y MÉTODOS}

El material utilizado es una muestra de acero AISI 316LVM, grado quirúrgico y cuya composición se registra en la Tabla 1, información obtenida mediante análisis químico por espectrometría de emisión óptica (o de chispa). Las probetas empleadas en los ensayos son circulares con un diámetro de $14 \mathrm{~mm}$ y 2,5 $\mathrm{mm}$ de espesor.

Tabla 1: Composición química del sustrato de acero

\begin{tabular}{|c|c|c|c|c|c|c|c|}
\hline Elemento & $\% \mathrm{Mn}$ & $\% \mathrm{C}$ & $\% \mathrm{Cr}$ & $\% \mathrm{Si}$ & $\% \mathrm{Mo}$ & $\% \mathrm{Fe}$ & $\% \mathrm{Ni}$ \\
\hline $\begin{array}{c}\text { AISI } \\
316 \mathrm{LVM}\end{array}$ & 1,57 & 0,03 & 17,25 & 0,76 & 2,64 & 63,34 & 14,41 \\
\hline
\end{tabular}

Para la evaluación de la resistencia a la corrosión en condición estática se utilizó un potenciostato galvanostato, Gamry modelo PCl-4. Se empleó la técnica de curvas de polarización Tafel. Los ensayos de corrosión electroquímica se realizaron a una temperatura de $37 \pm 0,2^{\circ} \mathrm{C}$, utilizando como electrolito solución fisiológica simulada conocida como solución de Hanks (Hanks balanced salt solution, Sigma) cuya composición química se detalla en la Tabla 2. Los ensayos se desarrollaron en función del tiempo a 0 y 196 horas. El montaje empleó una celda compuesta por un contraelectrodo de platino, un electrodo de referencia de plata/cloruro de plata $(\mathrm{Ag} / \mathrm{AgCl})$ y como electrodo de trabajo muestras de acero AISI 316LVM con un área expuesta de $1 \mathrm{~cm}^{2}$.Se realizaron las mediciones electroquímicas en duplicado.

Las muestras a evaluar fueron pulidas previamente hasta lograr una superficie de baja rugosidad, lo cual se logró mediante papeles abrasivosde SiC desde número 100 hasta 1200, y finalmente con suspensiones de alúmina $\left(\mathrm{Al}_{2} \mathrm{O}_{3}\right)$ de 1,0 y $0,05 \mu \mathrm{m}$, en disco rotatorio.

Las curvas de polarización fueron medidas a una hora de inmersión de las probetas en la solución de Hanks a una velocidad de barrido de $1 \mathrm{mV} / \mathrm{s}$, con voltajes de $-250 \mathrm{mV}$ hasta $+750 \mathrm{mV}$ respecto al potencial de corrosión (Ecorr). Los valores de velocidad de corrosión (Vcorr) se calcularon a partir de las pendientes de Tafel. Los valores de densidad de corriente de corrosión (Icorr) se determinaron a partir de un potencial de $\pm 250 \mathrm{mV}$ vs. Ecorr, en relación con las curvas de polarización anódicas.

Tabla 2: Composición química del electrolito (solución de Hanks).

\begin{tabular}{|c|c|}
\hline Compuesto & Concentración (g/l) \\
\hline $\mathrm{NaCl}$ & 8 \\
\hline $\mathrm{D}-\mathrm{Glucosa}$ & 1 \\
\hline $\mathrm{MgSO}_{2} 7 \mathrm{H}_{2} \mathrm{O}$ & 0,7 \\
\hline $\mathrm{Na}_{2} \mathrm{HPO}$ & 0,48 \\
\hline $\mathrm{KCl}$ & 0,4 \\
\hline $\mathrm{NaHCO}_{3}$ & 0,35 \\
\hline $\mathrm{CaC}_{12} \cdot \mathrm{H}_{2} \mathrm{O}$ & 0,18 \\
\hline $\mathrm{MgO}_{2} \cdot 6 \mathrm{H}_{2} \mathrm{O}$ & 0,08 \\
\hline $\mathrm{KH}_{2} \mathrm{PO}_{4}$ & 0,06 \\
\hline
\end{tabular}


Mediante un estereoscopio modelo Carl Zeiss con objetivo de 50X, se evaluó la superficie de las muestras de acero AISI 316LVM antes y después de someterlas a degradación en las solución de Hanks. El estudio determinó los cambios en la topografía de las probetas de acero posterior al ensayo electroquímico.

La identificación de los productos de corrosión generados en la superficie de los aceros se realizó mediante Difracción de Rayos X (DRX). El arreglo experimental en Difracción de Rayos X corresponde a Goniómetro PW3050/60 ( $(\theta / \theta)$, manejado bajo un sistema XPERT-PRO usando una radiación monocromática de Cu Ka $1,54 \AA$, operado a $40 \mathrm{kV}$ y $40 \mathrm{~mA}$ bajo condiciones de temperatura de $25^{\circ} \mathrm{C}$. El barrido sobre la superficie fue realizado desde $2 \theta=20,01^{\circ}$ hasta $2 \theta=99,99^{\circ}$ con un paso $2 \theta=0,02^{\circ}$ a un tiempo de barrido de 1 segundo. Las fases cristalinas se determinaron con la bases datos del equipo, además se utilizó el programa MAUD que permite el refinamiento de los difractogramas mediante el método Rietveld.

Para el análisis por visión artificial, se utilizó el software MATLAB® aplicando un tratamiento de imagen consistente en los siguientes pasos: (1) Convertir la imagen a escala de grises, (2) detección de bordes, (3) binarización, (4) dilación y (5) Erosión.

(1) La conversión a escala de grises se realiza la conversión de la imagen a una escala de 256 bits, donde 0 representa el color negro y 1 representa el color blanco, los demás bits entre 1 y 255 representa el degradado de color gris. La función que usa el toolbox de tratamiento de imágenes es la función rgb2gray. La función elimina el color y la saturación de cada pixel, pero dejando la iluminación de cada uno.

(2) En la detección de bordes de la imagen en escala de grises, la función usada para realizar la detección de bordes es la función edge, esta toma una imagen en escala de grises I como entrada, y retorna una imagen binaria (blanco y negro) del mismo tamaño de $I$ donde los 1 representan el lugar donde la función encontró bordes y 0 cualquier otro lugar. Este método utiliza uno de los siguientes filtros seleccionables: Sobel, Prewitt, Roberts y Canny.

Filtro Sobel: Matemáticamente, el operador utiliza dos kernels de $3 \times 3$ elementos para aplicar convolución a la imagen original para calcular aproximaciones a las derivadas, un kernel para los cambios horizontales y otro para las verticales. Si definimos $A$ como la imagen original, el resultado, que son las dos imágenes $G_{K}$ y $\mathrm{G}_{y}$ que representan para cada punto las aproximaciones horizontal y vertical de las derivadas de intensidades, es calculado como-se muestra en la ecuación 1.

$\mathrm{G}_{\mathrm{X}}=\left[\begin{array}{ccc}-1 & 0 & +1 \\ -2 & 0 & +2 \\ -1 & 0 & +1\end{array}\right] * \mathrm{~A} \quad y \quad \mathrm{G}_{\mathrm{Y}}=\left[\begin{array}{ccc}+1 & +2 & +1 \\ 0 & 0 & 0 \\ -1 & -2 & -1\end{array}\right] * A$

En cada punto de la imagen, los resultados de las aproximaciones de los gradientes horizontal y vertical pueden ser combinados para obtener la magnitud del gradiente, mediante la ecuación 2:

$$
\mathrm{G}=\sqrt{\mathrm{G}_{\mathrm{R}}^{2}+\mathrm{G}_{\mathrm{Y}}^{2}}
$$

Con esta información, es posible calcular también la dirección del gradiente, ecuación 3:

$$
\left(=\operatorname{arctang}\left(G_{-} y / G_{-} x\right)\right.
$$

donde, por ejemplo, $\Theta$ es 0 para bordes verticales con puntos más oscuros al lado izquierdo.

Filtro Prewitt: Matemáticamente, el filtro usa dos kernels de $3 \times 3$ los cuales son convolucionados con la imagen original para calcular aproximaciones a las derivadas, un kernel se usa para detectar los cambios horizontales y otro para los verticales. Si se define la matriz $A$ como la imagen fuente, y $G_{x} y G_{y}$ son dos imágenes las cuales en cada punto contienen las derivadas aproximadas horizontales y verticales las cuales son calculadas como:

$$
\mathrm{G}_{\mathrm{K}}=\left[\begin{array}{ccc}
-\mathbf{1} & \mathbf{0} & +\mathbf{1} \\
\mathbf{- 1} & 0 & +1 \\
-1 & \mathbf{0} & +1
\end{array}\right] * A \quad \mathbf{y} \quad \mathrm{G}_{\mathrm{y}}=\left[\begin{array}{ccc}
+1 & +1 & +1 \\
\mathbf{0} & \mathbf{0} & \mathbf{0} \\
-1 & -1 & -1
\end{array}\right] * A
$$

Donde $*$ denota la operación de convolución de 2 dimensiones. 
Dado que los kernels de Prewitt pueden ser descompuestos como un kernel aproximado y un kernel diferenciado, estos computan el gradiente con smoothing. Por ejemplo, $G_{\mathrm{z}}$ puede ser escrita como lo muestra la ecuación 5:

$$
\left[\begin{array}{lll}
-1 & 0 & +1 \\
-1 & 0 & +1 \\
-1 & 0 & +1
\end{array}\right]=\left[\begin{array}{l}
1 \\
1 \\
1
\end{array}\right]\left[\begin{array}{lll}
-1 & 0 & 1
\end{array}\right]
$$

La coordenada en $X$ se define como incremental hacia la derecha, y la coordenada $Y$ es definida como incremental hacia abajo. Es posible determinar la dirección del gradiente mediante la expresión de la ecuación 6:

$$
\left(=\operatorname{arctang} 2\left(G_{-} y_{s} G_{-} x\right)\right.
$$

Donde, por ejemplo $\Theta$ es 0 para un borde vertical el cual es más oscuro en el lado derecho.

Filtro Canny: El borde de una imagen puede apuntar en diferentes direcciones, por lo que el algoritmo de Canny utiliza cuatro filtros para detectar el horizontal, vertical y diagonal en los bordes de la imagen borrosa. El operador de detección de bordes (Prewitt, Sobel, por ejemplo) devuelve un valor para la primera derivada en la dirección horizontal ( $\left.G_{y}\right)$ y la dirección vertical $\left(G_{K}\right)$.

(3) La binarización se usa la función $B W$, esta convierte la imagen en escala de grises I en una imagen binaria. La salida de la función BW reemplaza los pixeles de la imagen de entrada con el brillo mayor al parámetro level con el valor de 1 (para blancos) y reemplaza todos los otros pixeles con el valor de 0 (para negros).

(4 - 5) La dilación y erosión. Inicialmente se realizó un tratamiento de imagen por medio de la función rgb2gray que convierte una imagen a color en una imagen a escala de grises, luego se utilizó la función edge para detectar los bordes de la figura y la función im2bw para convertir la imagen a binaria para su procesamiento.

Para el análisis de las imágenes se usaron las operaciones de imdilate e imerode funciones del toolbox de procesamiento de imágenes de MATLAB. La función imdilate dilata una imagen en formato binario por medio de la dilatación binaria, ecuación 7 , de $A$ por $B$ denotada ${ }^{A} \oplus \mathrm{B}$.

$\mathrm{A} \oplus \mathrm{B}=\left[\mathrm{z} \mid(\mathrm{B})_{\mathrm{z}} \cap \mathrm{A} \neq \emptyset\right]$

Donde $\widehat{B}$ es la reflexión del objeto estructurante de B, es decir, un grupo de pixeles localizados z, donde el elemento estructurante reflejado se superpone con píxeles de primer plano en A cuando se trasladan a $z$.

La función imerode realiza la erosión binaria de $A$ por $B$ denotada $A \ominus B$, ecuación 8 . En otras palabras son el conjunto de pixeles localizados $z$, donde el elemento estructurante trasladado a una localización z se superponen sólo con píxeles de primer plano en A

$\mathrm{A} \ominus \mathrm{B}=\left[\mathrm{z} \mid(\widehat{\mathrm{B}})_{\mathrm{z}} \subseteq \mathrm{A}\right]$

\section{RESULTADOS Y DISCUSIÓN}

\section{Análisis de degradación}

En la figura 1, se exponen las curvas potenciodinámicas para el acero AISI 316LVM evaluado a las 0 horas y 196 horasde inmersión en la solución fisiológica simulada (solución de Hanks). Como consecuencia de las curvas de polarización, anódica y catódica del acero inoxidable, es posible establecer la relación entre los mecanismos de polarización y el desarrollo de las reacciones en la superficie del material. También es posible determinar una diferencia importante en los potenciales de corrosión y las densidades de corriente de corrosión (Sabine, 2010; Shahryari, 2008) Para el sistema evaluado a 0 horas de inmersión, se observa con mayor estabilidad y menor energía cinética corrosiva. En relación con el sistema evaluado a 196horas, se advierte una leve disminución del potencial de corrosión y un aumento gradual en la densidad de corriente de corrosión. 
Para las dos situaciones estudiadas, es posible determinar una zona clara de pasivación y transpasivación. Es importante notar la modificación en la pasivación del acero inoxidable a las 196 horas, condición impuesta por la solución fisiológica simulada. A 0 horas el mecanismo de polarización que prevalece pareciera ser únicamente por transferencia de carga, mientras que para el caso de 196 horas predomina un fenómeno difusivo (Langer, 1990; Tjong, 1990).

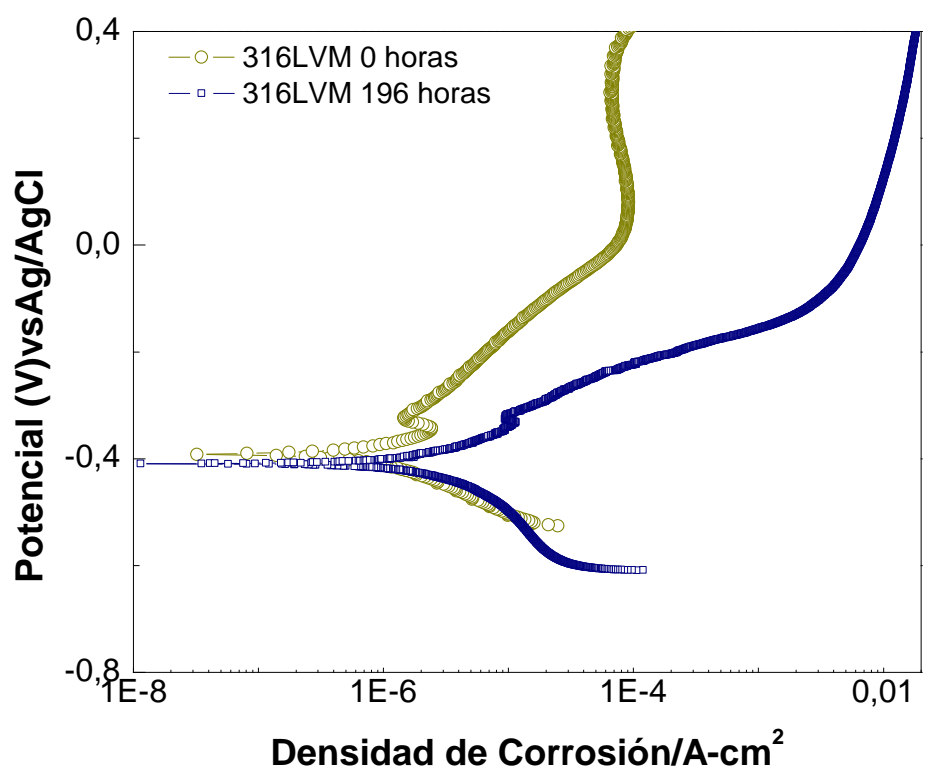

Fig. 1: Curvas potenciodinámicas del acero AISI 316LVM a 0 y 196 horas de inmersión en solución de Hanks.

En la Tabla 3, se registran los parámetros calculados a partir de las curvas de polarización en el rango de los $\pm 250 \mathrm{mV}$. Se observan diferencias en potenciales de reducción, siendo más noble el material316LVM a las 0 horas y un potencial de corrosión más activo para la aleación luego de la exposición las 196 horas. Además, los valores de velocidad y corriente de corrosión para la aleación evaluado a 0 horas tienen un comportamiento similar que la aleación a las 196 horas, generando parámetros de corrosión con mínimos valores para ambos casos.

Tabla 3: Parámetros del acero 316 LVM, hallados con las curvas de polarización a las 0 y 196 horas.

\begin{tabular}{|c|c|c|}
\hline Parámetro & 0 horas & 196 horas \\
\hline $\begin{array}{c}\text { Pendiente Anódica } \\
(\text { V/década) }\end{array}$ & $132,11 \times 10^{-3}$ & $196,54 \times 10^{-3}$ \\
\hline $\begin{array}{c}\text { Pendiente Catódica } \\
(\text { V/década) }\end{array}$ & $-36,21 \times 10^{-3}$ & $-211,28 \times 10^{-3}$ \\
\hline $\begin{array}{c}\text { Corriente de Corrosión } \\
(\mu \mathrm{A})\end{array}$ & 1,74 & 6,39 \\
\hline $\begin{array}{c}\text { Potencial de corrosión } \\
(\mathrm{mV})\end{array}$ & $-391,11$ & $-411,42$ \\
\hline $\begin{array}{c}\text { Velocidad de Corrosión } \\
(\mathrm{mpy})\end{array}$ & 1,69 & 5,36 \\
\hline
\end{tabular}

\section{Análisis por visión artificial}

Una vez transcurridos los tiempos de inmersión de las muestras de acero AISI 316LVM en la solución de Hanks, se tomaron las correspondientes imágenes topográficas; en la figura 2 se observan dichas imágenes. La figura 2a corresponde a la imagen en el estado inicial del estudio (antes de la inmersión). Las figuras $2 \mathrm{~b}$ y $2 \mathrm{c}$, permiten visualizar los resultados finales en relación con los tiempos de inmersión en la solución fisiológica simulada (0 h y 160 h), respectivamente. 


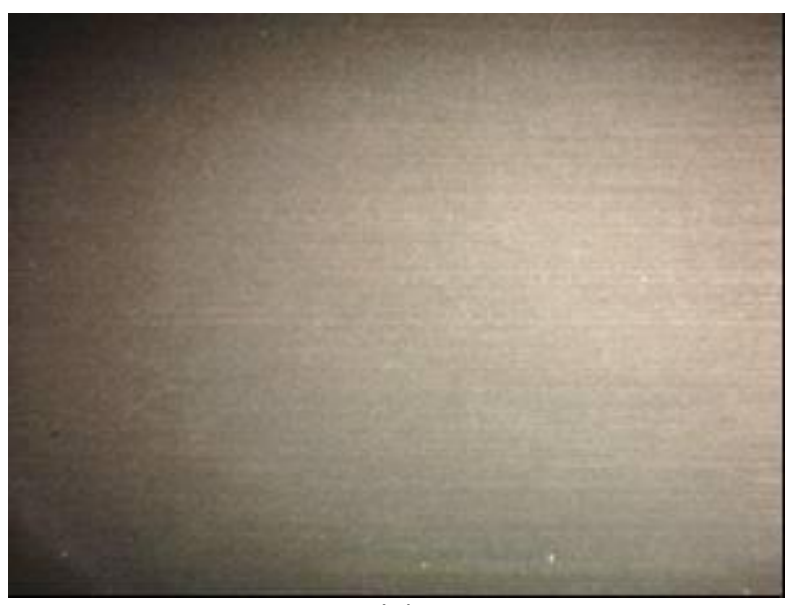

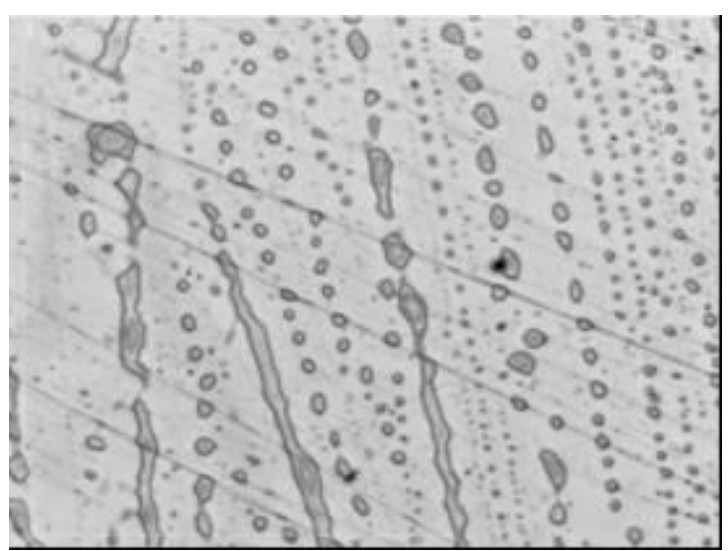

(b)

(a)

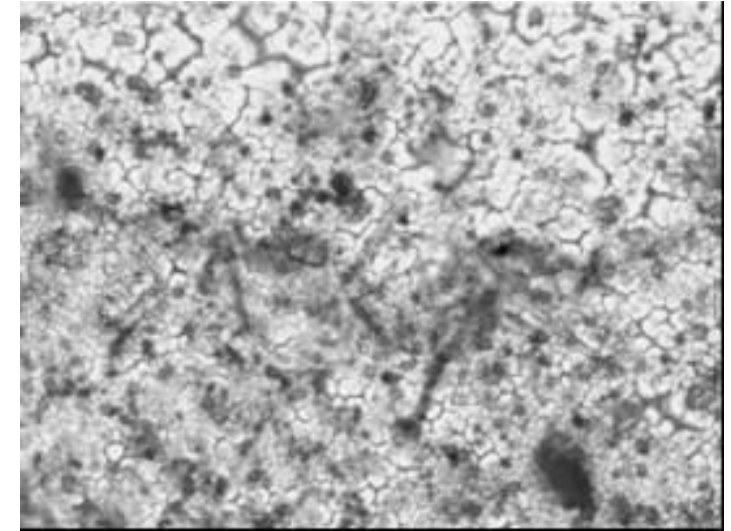

(c)

Fig. 2: Imágenes superficiales para muestras a) Acero inoxidable 316LVM, sin exposición: b) Acero 316LVM, evaluado a 0 horas de inmersión; c) Acero 316LVM, evaluado a 196 horas de inmersión.

Para el análisis gráfico por comparación se hizo a partir de la toma de las imágenes de la totalidad de muestras evaluadas con el propósito de encontrar las diferencias. Se eligió como referencia la imagen del material sin degradación (figura 2a), posteriormente se seleccionaron las imágenes obtenidas de las muestras a 0 horas y 160 horas de inmersión en la solución de Hanks. Los porcentajes de corrosión calculados mediante el análisis de visión artificial se ven corroborados con respecto a los resultados encontrados de acuerdo con las curvas de polarización anódica. Según los resultados obtenidos, la aplicación de la metodología por visión artificial permite evaluar los procesos de corrosión que se llevan a cabo sobre las muestra de acero ASI 316LVM cuando entran en contacto con una solución fisiológica simulada o de Hanks.

La figura 3a muestra el resultado de la diferencia entre las imágenes, de la probeta sin someterse a la degradación (2a) y luego de aplicar el ensayo electroquímico a las 0 horas (2b). Esta diferencia se obtiene a través de el toolbox de procesamiento de imágenes que contiene un conjunto de funciones de los algoritmos que trabajan con imágenes binarias, trasformaciones geométricas, morfología y manipulación de color que junto con las funciones ya integradas en Matlab permitiendo realizar los análisis y trasformaciones de imágenes en el dominio de la frecuencia (trasformada de Fourier).

Analizando la figura $3 a$ se observa que toda la superficie del material se ve afectado al entrar en contacto con la solución de Hanks. Con ayuda del software MATLAB® se determinó el factor de la degradación presente, mediante el empleo de métodos estadísticos de reconocimiento de color y basándose en métodos inteligentes de clasificación y de visión de máquina. Como respuesta, el software muestra las imágenes de las figuras $3 \mathrm{~b}$ y $3 \mathrm{c}$.Contrastando las zonas de corrosión halladas en la figura 3a se determinaron el número de pixeles que representan las zonas atacadas por la solución fisiológica simulada. De esta forma se determinó el factor de corrosión el cual, para esta prueba, fue de16,39\%. 


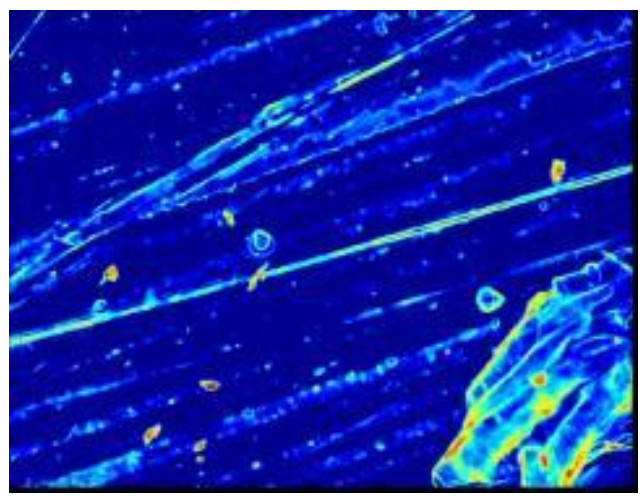

(a)

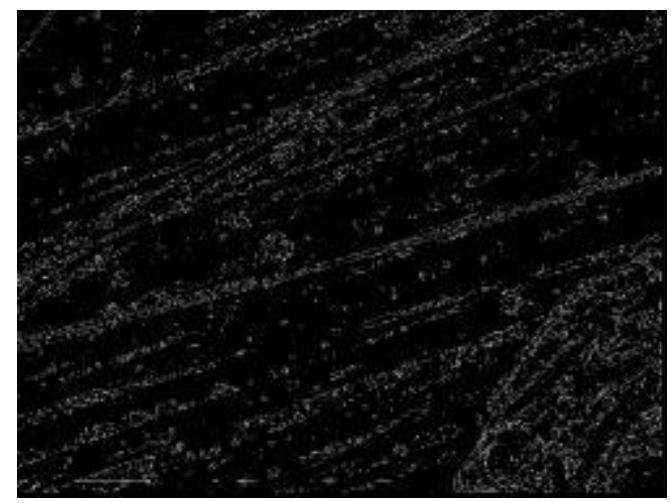

(b)

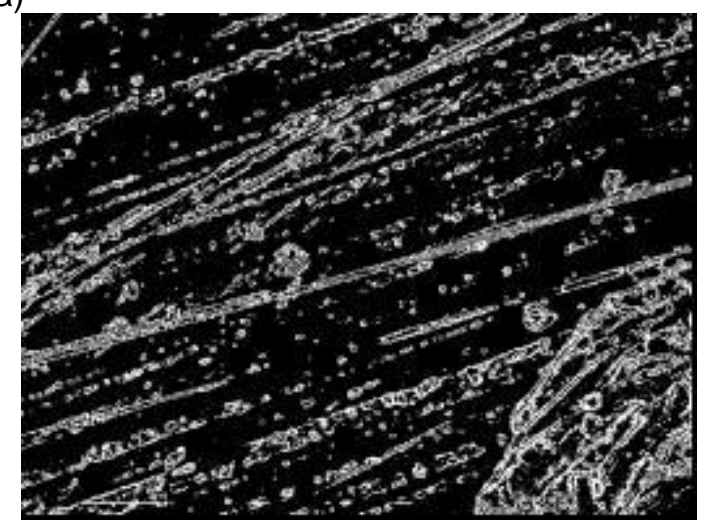

(c)

Fig. 3: a) Diferencia absoluta entre las imágenes (Sin y con ataque electroquímico) a las 0 horas.

b) Detección binaria de bordes de la diferencia absoluta. c) Bordes acentuados mediante un proceso de dilatación.

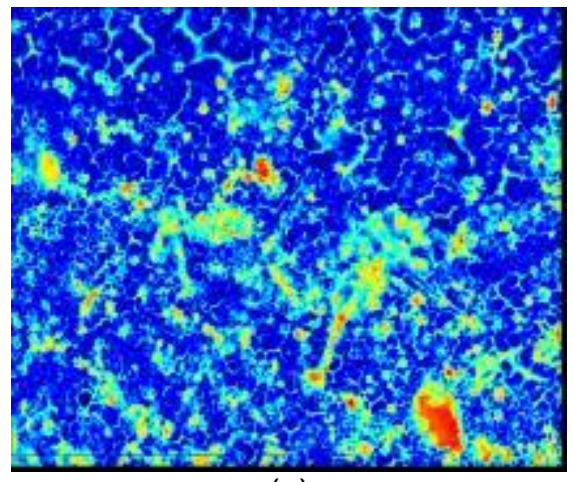

(a)

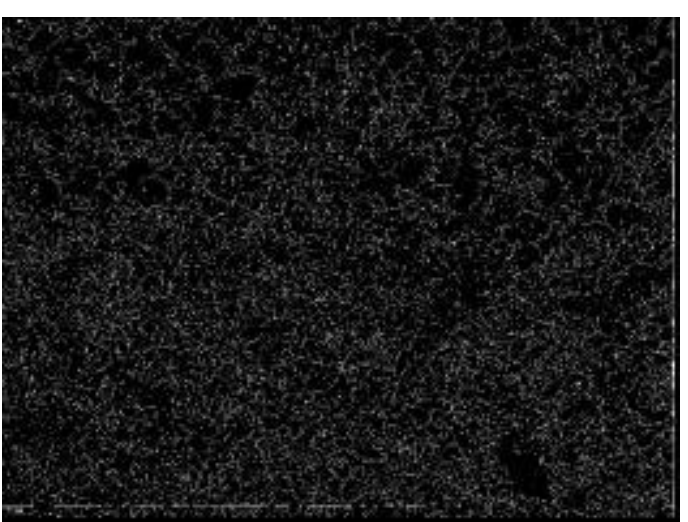

(b)

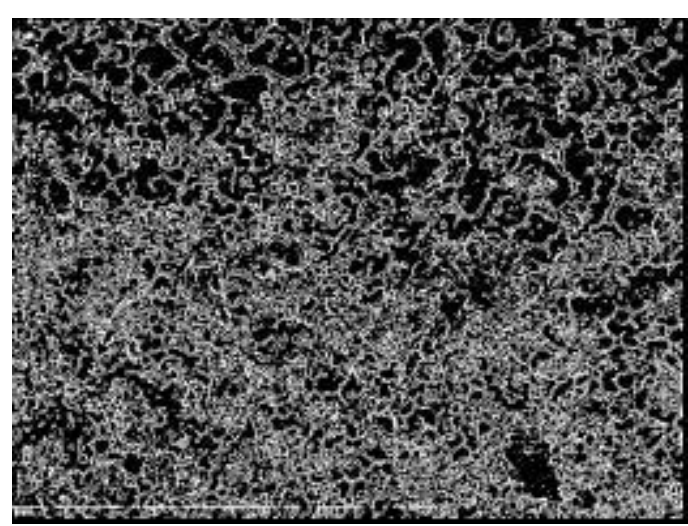

(c)

Fig. 4: a) Diferencia absoluta entre las imágenes (Sin y con ataque electroquímico) con exposición a 196 horas. b) Detección binaria de bordes de la diferencia absoluta. c) Bordes acentuados mediante un proceso de dilatación. 
Continuando con el análisis por visión artificial, en la figura 4 se observan los resultados del desempeño del material después de las 196 horas de exposición al ambiente agresivo. Se aplicó el mismo procedimiento como en el caso anterior, con ayuda de MATLAB®, para el procesamiento de las imágenes. Se obtuvo una imagen que genera mayor cantidad de pixeles asociados al proceso de degradación, esto indica un aumento en el factor de corrosión superficial calculado en un $54 \%$.

\section{Identificación de productos de corrosión}

Mediante el difractograma de la figura 5 , se identifican los productos de corrosión del acero inoxidable 316LVM después de los ensayos de corrosión a 0 horas y 196 horas, respectivamente. Se observan algunos picos característicos de los productos de corrosión presentes en la superficie del material como lo son: óxido de cromo, óxido de hierro y espinela de hierro-silicio. Dado que el acero inoxidable presenta dos características importantes desde el punto de vista de la resistencia a la corrosión, la capacidad de pasivación y la estabilidad termodinámica, para el ensayo a las 0 horas se observan picos característicos de la fase austenítica como la predominante. Sin embargo, se observan algunos picos correspondientes a los productos de corrosión típicos del acero inoxidable. Al comparar con los resultados obtenidos a las 196 horas se observa que los picos correspondientes a los productos de corrosión han aumentado en intensidad, indicando mayor presencia de óxido de hierro. Esta información se corroboró con los resultados del análisis de visión artificial, en donde a las 196 horas se obtiene un alto factor de degradación representado como porcentaje de productos de corrosión.

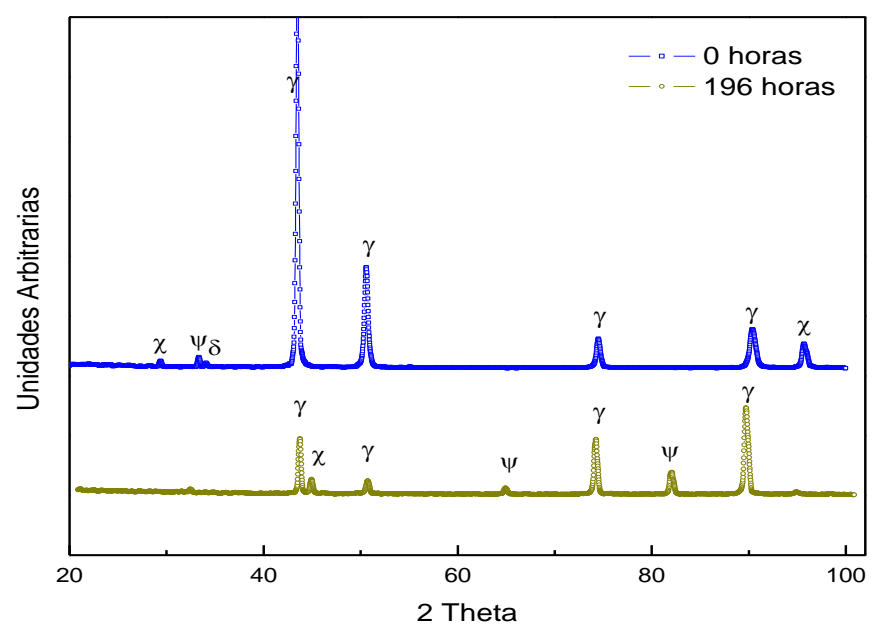

Fig. 5: Difractogramas del acero inoxidable 316LVM. $\gamma=$ austenita; $\chi=\mathrm{Cr}_{2} \mathrm{O}_{3} ; \delta=\mathrm{Fe}_{2} \mathrm{SiO}_{4} ; \psi=\mathrm{Fe}_{2} \mathrm{O}_{3}$.

\section{CONCLUSIONES}

Existen herramientas y paquetes software cuya finalidad principal son las aplicaciones industriales, tal es el caso de el Toolbox Image de MATLAB®. La información que un sistema de visión artificial logra proveer es particularmente aplicable en la evaluación de la degradación sufrida por soluciones fisiológicas simuladas en muestras de acero AISI 316 LVM y la correspondiente determinación del factor de corrosión superficial.

En el área de biomateriales, resulta de vital importancia conocer la vida útil de cada material o implante utilizado en el cuerpo humano con el fin de establecer el periodo de tiempo en el cual debería ser este reemplazado, y así, prevenir posibles fallos causados por el cambio en las propiedades del material debido al medio en el que está trabajando.

La aplicación de la visión artificial en la determinación del factor de corrosión permite: (1) simplicidad en el procedimiento. (2) posibilidad de realizar pruebas bajo diferentes medios de trabajo, personalizados según la necesidad, como por ejemplo fluido en movimiento o estático. (3) bajo costo y velocidad de análisis y (4) esta tecnología se puede combinar con otras técnicas convencionales para reforzar los resultados encontrados. 
Del estudio se observó que tanto en el análisis gráfico (visión artificial) y corroborado con el análisis electroquímico, la corrosión se hace mucho más notoria a elevados tiempo de exposición. Estos resultados son de vital importancia, porque permitieron dar a conocer la relación existente entre la reacción y el cambio topográfico del material a emplear como implante quirúrgico bajo las condiciones impuestas por fluidos fisiológicos simulados.

Se desarrolló un software capaz de identificar el factor de degradación en una muestra que fue sometida a diferentes medios o ambientes degradantes, por medio de visión artificial, además permitió establecer el factor y el tipo de degradación presente en las muestras.

\section{REFERENCIAS}

Bonfield, W., Mechanical properties of bone. Biomaterials, 2, 251-252 (1981).

Bou-Saleh, Z., Shahryari, A. y Omanovic, S. Enhancement of corrosion resistance of a biomedical grade 316LVM stainless steel by potentiodynamic cyclic polarization. Thin Solid Films, 515, 4727-4737 (2007).

Bordji, K. y otros siete autores. Cytocompatibility of Ti-6Al-4V and Ti-5AI-2.5Fe alloys according to three surface treatments, using human fibroblasts and osteoblasts. Biomaterials, 17, 929-940 (1996).

Brune, D., y Hultquist G. Corrosion of a stainless steel with low nickel content under static conditions, Biomaterials, 6, 265-268 (1985).

Chmiel, M., Słowiński, M. y Dasiewicz, K., Application of computer vision systems for estimation of fat content in poultry meat, Food Control, 22(8), 1424-1427 (2011).

Cook, S.D., The in vivo performance of 250 internal fixation devices; a fellow up study, Biomaterials, 8, 177184 (1986).

De Mello, J.D.B. y De S. Balsamo, P.S., Comportamiento Tribológico de Aceros Inoxidables para Cubertería, Información Tecnológica, 17 (6), 57-62 (2006).

Dutta, S., Das, A., Barat, K., y Himadri, R. Automatic characterization of fracture surfaces of AISI 304LN stainless steel using image texture analysis, Measurement , 45, 1140-1150 (2012).

Geetha, M. y otros dos autores, Ti based biomaterials, the ultimate choice for orthopaedic implants, Progress in Materials Science, 54, 397-425 (2009).

German, S., Brilakis, I. y DesRoches, R. Rapid entropy-based detection and properties measurement of concrete spalling with machine vision for post-earthquake safety assessments, Advanced Engineering Informatics, 26, 846-858 (2012).

Infaimon, C., Visión artificial aplicada a la industria (2010),

http://www.jcee.upc.es/JCEE2010/pdf_ponencies/PDFs/25_11_10/INFAIMON-Vision\%20artificial.pdf.

Acceso: 27 de agosto (2012)

Jacobs, J. y otros seis autores, J. Clinical Orthopedics and Related Research, 358, 1999, 173-180 (1999).

Langer, R., Cima, L.G., Tamada, J.A. y Wintermantel, E. Future directions in biomaterials, Biomaterials, 11, 738-745 (1990).

López, D.A., Durán, A. y Ceré, S., Caracterización superficial de acero inoxidable AISI 316L en contacto con solución fisiológica simulada, Actas del Congreso CONAMET/SAM, 256-260, La Serena, Chile, 3 al 5 de noviembre (2004).

Moreda, G.P., Muñoz, M.A., Ruiz-Altisent, M. y Perdigones, A., Shape determination of horticultural produce using two-dimensional computer vision - A review, Journal of Food Engineering, 108(2), 245-261 (2012).

Park, J.B. The Biomedical Engineering Handbook, 2a edición, 45-56. CRC Press, USA (1999)

Pourbaix, M. Electrochemical corrosion of metallic biomaterials, Biomaterials, 5, 122-134 (1984). 
Rojas, T.V., Sanz, W. y Arteaga, F. Sistema de visión por computadora con la transformada de Hough. revista ingeniería UC, 15(1), 77-87 (2008).

Sabine, B., Fung Ang, S., y Schneider, G.A.On the mechanical properties of hierarchically structured biological materials. Biomaterials, 31, 6378-6385 (2010).

Samuel, S., Nag, S., Scharf, T. W. y Banerjee, R. Wear resistance of laser-deposited boride reinforced Ti$\mathrm{Nb}-\mathrm{Zr}-\mathrm{Ta}$ alloy composites for orthopedic implants, Materials Science and Engineering: C, 28, 414-420 (2008).

Shahryari, A., Omanovic, S. y Szpunar, J.A.Electrochemical formation of highly pitting resistant passive films on a biomedical grade 316LVM stainless steel surface, Materials Science and Engineering: C, 28, 94-106 (2008).

Tapash, R., Rautray, R. y Kyo-Han, K. Ion implantation of titanium based biomaterials, Progress in Materials Science, 56, 1137-1177 (2011).

Tjong, S.C., Electron microscope observations of phase decompositions in an austenitic Fe-8.7Al-29.7Mn1.04C alloy. Materials Characterization, 24,275-292 (1990).

Venugopalan, R. y Gaydon, J., A Review of Corrosion Behaviour of Surgical Implant Alloys, Perkin Elmer Instruments, 99-107, Princeton, USA (2001).

Woodman, J., Black, J. y Nunamaker, D., J. Biomed. Mater. Res: 17(1), 655-658 (1983).

Zion, B., The use of computer vision technologies in aquaculture - A review, Computers and Electronics in Agriculture, 88, 125-132 (2012). 
